\title{
La liberté de religion dans le contentieux européen de l'éloignement des étrangers : entre réalisme et exigence d'effectivité
}

\section{Mustapha Afroukh}

\section{OpenEdition \\ Journals}

Édition électronique

URL : http://journals.openedition.org/rdr/860

DOI : $10.4000 /$ rdr. 860

ISSN : 2534-7462

Éditeur

Presses universitaires de Strasbourg

Édition imprimée

Date de publication : 3 mai 2017

Pagination : 111-124

ISBN : 978-2-86820-973-3

ISSN : 2493-8637

\section{Référence électronique}

Mustapha Afroukh, «La liberté de religion dans le contentieux européen de l'éloignement des

étrangers : entre réalisme et exigence d'effectivité », Revue du droit des religions [En ligne], 3 | 2017, mis en ligne le 03 février 2020, consulté le 19 novembre 2020. URL : http://journals.openedition.org/rdr/ 860 ; DOI : https://doi.org/10.4000/rdr.860

\section{(c) (†) (8)}

La revue du droit des religions est mise à disposition selon les termes de la Creative Commons Attribution - Pas d'Utilisation Commerciale 4.0 International - CC BY-NC 4.0. 


\section{LA LIBERTÉ DE RELIGION DANS LE CONTENTIEUX EUROPÉEN DE L'ÉLOIGNEMENT DES ÉTRANGERS: ENTRE RÉALISME ET EXIGENCE D'EFFECTIVIIÉ}

\section{Mustapha AFROUKH}

Université de Montpellier, Institut de droit européen des droits de l'homme (IDEDH)

\section{RÉSUMÉ}

Dans un contexte international favorisant les départs de nombreux migrants persécutés en raison de leur appartenance religieuse, la question se pose de savoir si la liberté de religion peut être invoquée, dans le contentieux des étrangers, pour faire obstacle à l'exécution d'une mesure d'éloignement. La réponse donnée par la Cour européenne des droits de l'homme oscille entre conservatisme et audace. Si elle se refuse toujours à reconnaître un effet extraterritorial autonome à la liberté de religion protégée à l'article 9 de la Convention européenne, elle n'hésite plus à prendre en compte les risques de persécution religieuse.

\section{ABSTRACT}

In an international context which results in the departure of many migrants persecuted on the basis of their religious affiliation, the question arises whether freedom of religion could be relevant as a barrier to the implementation of any expulsion measure in aliens litigation. The answer given by the European Court of Human Rights stands between conservatism and audacity. While the Court still refuses to admit an autonomous extraterritorial effect on the freedom of religion protected by Article 9 of the European Convention, it no longer hesitates to take into account persecution risks on religious grounds. 
$\mathrm{U}$ n État partie à la Convention européenne des droits de l'homme peut-il éloigner un individu vers un État où l'exercice de sa liberté de religion (art. 9) risque d'être limité? La question, bien que technique, soulève des enjeux considérables tenant aux tentatives d'arrimage du droit d'asile à la Convention européenne ${ }^{1}$. La difficulté est bien connue : si la Cour européenne applique la Convention aux demandeurs d'asile et insiste tout particulièrement sur leur situation d'extrême vulnérabilité, elle ne cesse de rappeler que la Convention et ses protocoles ne garantissent pas le droit de rechercher et de demander l'asile ${ }^{2}$. Aussi, souligne-t-elle que son rôle n'est pas « d'examiner elle-même les demandes d'asile ou de contrôler la manière dont les États remplissent leurs obligations découlant de la convention de Genève relative au statut des réfugiés $»^{3}$. La situation est toute autre en droit de l'Union européenne avec l'existence d'un régime européen de l'asile constitué de plusieurs textes de droit dérivé et fondé sur une application intégrale de la convention de Genève, qualifiée de « pierre angulaire du régime juridique international de protection des réfugiés $»^{4}$. S'agissant du système européen de protection des droits de l'homme, les liens entre le droit d'asile et la Convention européenne ne peuvent être envisagés qu'à la lumière de la jurisprudence relative à l'éloignement des étrangers. Toute la difficulté pour la Cour est de prendre en compte la situation des demandeurs d'asile sans dépasser les limites de son office.

Sans doute est-il utile de rappeler, à titre liminaire, que la lettre de la Convention ne nous renseigne guère sur son applicabilité dans le domaine du droit des étrangers. En effet, le texte initial de la Convention était silencieux sur ce point. Aussi, soucieuse d'effectivité, la Cour a développé une jurisprudence audacieuse et particulièrement constructive pour combler ce vide juridique. On songe évidemment au mécanisme de la «protection par

1. Chetail V., «Le droit des réfugiés à l'épreuve des droits de l'homme : bilan de la jurisprudence de la Cour européenne des droits de l'homme sur l'interdiction du renvoi des étrangers menacés de torture et de traitements inhumains et dégradants », RBDI 2004/1, p. 155.

2. CEDH, 30 oct. 1991, Vilvarajah et a. c/ Royaume-Uni, \& 102, série A n 215.

3. Pour une affirmation récente, CEDH, Gde ch., 23 mars 2016, nº 43611/11, F.G. c/ Suède, $\S 117$.

4. V., parmi d'autres, CJUE, 2 mars 2010, aff. C-175/08, Aydin Abdulla, Rec. p. I-1493, pt. 53 et CJUE, 17 juin 2010, aff. C-31/09, Bolbol, pt. 38. Selon J.-Ch. MARTin, « ce qui caractérise la relation entre la Convention de 1951 et les régimes européens de protection [...], c'est la primauté de la Convention de 1951 à laquelle tous les Etats membres de l'Union sont parties » ( L La cohérence des régimes de protection dans l'Union européenne », in Millet-Devalle A.-S. (dir.), L'Union européenne et la protection des migrants et des réfugiés, Paris, Pedone, 2010, p. 191). 
ricochet » qui permet d'étendre la protection de certains droits et libertés à des situations non visées par la Convention. Alors que le droit à ne pas être expulsé ou extradé ne figure pas comme tel au nombre des droits et libertés garantis par la Convention, il est établi depuis l'important arrêt Soering que des risques réels et sérieux de traitements contraires à l'article $3^{5}$ dans l'État de destination rendent l'exécution de la mesure d'éloignement constitutive d'une violation de la Convention. L'originalité de cette jurisprudence est double : d'abord, elle conduit le juge européen à se prononcer sur une violation virtuelle de la Convention. Comme l'a fort justement souligné Jennifer Marchand, " on suppute le risque d'une future violation de l'article 3 [...]. Si la Cour retient la violation avant même qu'elle se réalise - dès lors que l'expulsion n'a pas été mise en ouvre - l'atteinte aux droits garantis n'en apparaît pas moins réelle ${ }^{6}$. Tout bien considéré, pèse sur les États une obligation de prévenir les violations de l'article 3 en cas d'éloignement d'un étranger. Ensuite et surtout, la jurisprudence Soering confère à l'article 3 une portée extraterritoriale de la Convention en ce que le juge européen est nécessairement amené à « apprécier la situation dans le pays de destination à l'aune des exigences [de la Convention] $»^{7}$.

Dans l'arrêt Soering, la Cour s'appuie fortement sur la singularité de l'article 3 dans le corpus européen des droits de l'homme pour justifier une telle extension de la protection conventionnelle : « [cette disposition] ne ménage aucune exception et l'article 15 ne permet pas d'y déroger en temps de guerre ou autre danger national. Cette prohibition absolue, par la Convention, de la torture et des peines ou traitements inhumains ou dégradants montre que l'article 3 consacre l'une des valeurs fondamentales des sociétés démocratiques qui forment le Conseil de l'Europe » (§ 88). L'on peut s'interroger sur le fait de savoir si cet effet extraterritorial bénéficie également à la liberté de religion protégée à l'article 9 de la Convention. La question est d'autant plus légitime que le juge européen semble faire dépendre cet effet de l'importance des droits en cause. Or, si ce droit ne fait pas partie de la

5. « Nul ne peut être soumis à la torture ni à des peines ou traitements inhumains ou dégradants. »

6. Marchand J., « Prévention et dissuasion dans la jurisprudence de la Cour européenne des droits de l'homme », RFDA 2014, p. 1150.

7. CEDH, Gde ch., 7 juill. 1989, n 14038/88, Soering c/ Royaume-Uni, \& 91. Dans cette situation, l'effet extraterritorial de la Convention « appréhende des situations se déroulant ou ayant pris naissance sur le territoire d'un État et/ou qui produisent leurs effets hors du territoire de l'État auteur » (LAgrange E., «L'application de la Convention de Rome à des actes accomplis par les États parties en dehors du territoire national », RGDI publ. 2008, p. 525). 
liste des droits indérogeables, son aspect interne qui renvoie aux croyances et aux convictions de l'individu ne peut, en aucun cas, «nous être ôté par une puissance supérieure ${ }^{8}$. Seule la liberté de manifester sa religion ou ses convictions, c'est-à-dire son aspect externe, son exercice concret, peut faire l'objet de restrictions selon le paragraphe 2 de l'article 9. En tant qu'elle protège le for intérieur, la liberté de religion peut être considérée comme une liberté absolue. Notons surtout que la liberté de religion constitue un droit fondamental dans la jurisprudence européenne. C'est ce dont témoigne la formule célèbre de l'arrêt Kokkinakis selon laquelle « la liberté de pensée, de conscience et de religion [...] représente l'une des assises d'une "société démocratique" au sens de la Convention ${ }^{9}$. La Cour a également pu juger que la liberté de religion est «dans sa dimension religieuse, l'un des éléments les plus vitaux contribuant à former l'identité des croyants et leur conception de la vie $»^{10}$, soulignant ainsi l'objet de la liberté de religion dans l'accomplissement de l'individu en tant que créature spirituelle, donc de son caractère essentiel pour la personne humaine.

Cet appel à la fondamentalité ne revêt pas une simple force rhétorique, invoquer l'importance d'un droit dans le standard de société démocratique permet souvent de lui conférer une protection spécifique. En outre, l'extension du raisonnement de la Cour dans l'affaire Soering à la liberté de religion s'inscrirait dans la logique de la convention de Genève de 1951 qui prévoit que le statut de réfugié peut être reconnu à toute personne répondant à la définition de l'article $1^{\text {er }}$, A, 2 qui craint de subir des persécutions en raison de ses opinions politiques, de son appartenance religieuse, de sa race, de sa nationalité ou de son appartenance à un certain groupe social. Allant plus loin, l'article 10 de la directive dite « Qualification » 2004/83/CE du 29 avril 2004 prévoit même que « la notion de religion recouvre, en particulier, le fait d'avoir des convictions théistes, non théistes ou athées, la participation à des cérémonies de culte privées ou publiques, seul ou en communauté, ou le fait de ne pas y participer, les autres actes religieux ou expressions d'opinions religieuses, et les formes de comportement personnel ou communautaire fondées sur des croyances religieuses ou imposées par ces croyances », ce qui explique que la Cour de justice développe ici une interprétation autonome et audacieuse ${ }^{11}$, on y reviendra.

8. E. Kant, cité par LauPies F., La liberté, Paris, PUF, « Que sais-je? », 2004, p. 103.

9. CEDH, 25 mai 1993, n 14307/88, Kokkinakis c/ Grèce, § 31.

10. CEDH, 20 sept. 1994, n 13470/87, Otto-Preminger-Institut c/ Autriche, § 47.

11. CJUE, 5 sept. 2012, aff. jointes C-71/11 et C-99/11, Bundesrepublik Deutschland c/ Y et Z, AJDA 2012, p. 2267, chron. Aubert M., Broussy E., CASSAgnabere H. ; RDP 2013, p. 707, note SCHAHMANECHE A. 
Ces dernières années, le contexte international a favorisé des exodes de masse et des départs de nombreux migrants persécutés en raison de leur appartenance religieuse. L'exemple dramatique des chrétiens d'Irak pourchassés par l'armée de Daesh est topique à cet égard. Conscients des potentialités qu'offre la jurisprudence Soering, de nombreux requérants n'hésitent plus à saisir le juge européen pour contester leur éloignement en invoquant des risques de persécution religieuse. C'est ainsi que nous sont livrés des enseignements sur le traitement réservé à la liberté de religion dans le contentieux de l'éloignement des étrangers. De toute évidence, la Cour refuse de s'ériger en juge européen de l'asile, mais elle n'ignore pas les risques de persécution religieuse au titre de sa jurisprudence Soering. Pour le dire autrement, le refus de conférer un effet extraterritorial à la liberté de religion (1) ne signifie pas que la liberté de religion n'est pas protégée, la jurisprudence récente ouvrant timidement la voie à une garantie indirecte de cette liberté dans le contentieux de l'éloignement (2). La jurisprudence étudiée est intéressante parce qu'elle illustre à merveille la tension entre audace et prudence du juge. Le récent arrêt F. G c/ Suède $e^{12}$ constitue d'ailleurs le dernier avatar particulièrement emblématique de cette tension jurisprudentielle : il souffle le chaud et le froid, oscillant entre innovation et conservatisme.

\section{LE REFUS DE CONSACRER L'EFFET EXTRATERRITORIAL DE LA LIBERTÉ DE RELIGION}

Pétrie de réalisme (1.1), la solution refusant d'étendre mécaniquement l'effet extraterritorial de la CEDH à la liberté de religion accrédite l'idée d'une hiérarchisation des droits et libertés au sein de l'ordre conventionnel (1.2).

\subsection{UNE SOLUTION RÉALISTE}

La question de l'invocabilité de la liberté de religion dans le contentieux de l'éloignement a été posée pour la première fois à l'occasion d'une affaire Katani c/ Allemagne dans laquelle les requérants alléguaient qu'en cas d'expulsion vers la Géorgie, ils risquaient de subir des mauvais traitements en raison de leur appartenance à la religion yézidie ${ }^{13}$. Alors qu'ils invoquaient directement l'article 9, la Cour oppose un raisonnement à tout le moins

12. CEDH, Gde ch., 23 mars 2016, précit.

13. CEDH, déc. 31 mai 2001, n 67679/01, Katani c/ Allemagne. 
équivoque : " eu égard à ce qui a été relevé ci-dessus [grief tiré d'une violation de l'article 3 considéré comme manifestement mal fondé], la Cour note qu'elle n'est pas appelée à se prononcer sur la question de savoir si l'article 9 de la Convention peut, dans certains cas, impliquer la responsabilité d'un État qui s'apprête à expulser une personne vers un autre État contractant». Inutile de dire ici que l'article 9 est éclipsé par l'article 3 qui est le vecteur principal de la protection par ricochet ${ }^{14}$. Un autre cas particulièrement significatif est sans conteste la décision d'irrecevabilité $Z$. et T. c/ Royaume-Uni ${ }^{15}$. Saisi par des ressortissantes pakistanaises de confession chrétienne menacées d'expulsion vers le Pakistan, le juge européen estime que la reconnaissance de l'application extraterritoriale de l'article 9 reviendrait « [à imposer] aux États parties une obligation d'agir effectivement en tant que garants indirects de la liberté de religion et de culte pour le reste du monde». L'accent mis sur les obligations internationales issues de la convention des Nations unies relative au statut des réfugiés révèle une nette hostilité de la Cour européenne aux tentatives d'arrimage du droit d'asile à la Convention. En ce sens, sont rappelées les limites de l'office de la Cour qui n'est pas juge de l'asile. La force des motifs avancés par le juge laisse penser que la liberté de religion ne peut pas faire l'objet d'un examen séparé. Elle n'est protégée que si les persécutions alléguées exposent l'étranger à un risque sérieux pour sa liberté ou son intégrité physique ${ }^{16}$. L'effet extraterritorial conféré à d'autres dispositions devrait suffire à empêcher que « des droits humains fondamentaux, tels que ceux qui sont garantis par la Convention, pourraient être soit grossièrement violés, soit entièrement supprimés ${ }^{17}$. La logique réaliste atteint son paroxysme lorsque la décision indique « [qu'on] ne saurait exiger qu'un État contractant procédant à une expulsion ne renvoie un étranger que dans un pays où la situation est pleinement et effectivement conforme à chacun des droits et libertés garantis par la Convention ${ }^{18}$. On ne peut alors se

14. Dans le même sens, CEDH, déc. 11 mars 2003, n 64599/01, Razaghi c/ Suède : "En ce qui concerne le droit du requérant à la liberté de religion, la Cour observe que, dans la mesure où aucune conséquence présumée en Iran de la conversion de la requérante au christianisme n'atteint le niveau de traitement prohibé par l'article 3 de la Convention [...]. La Cour estime que l'expulsion du requérant ne peut engager séparément la responsabilité du gouvernement suédois en vertu de l'article 9 de la Convention. » [trad. par nous].

15. CEDH, déc. 28 févr. 2006, n 27034/05, Z. et T. c/ Royaume-Uni, AJDA 2006, p. 1712, chron. Flauss J.-F. Selon les requérantes, les obliger à dissimuler leur adhésion au christianisme reviendrait à une négation totale de leur droit à la liberté de religion.

16. Pour une illustration, V. l'arrêt M. E. c/ France du 6 juin 2013 (persécutions subies par des chrétiens coptes en Égypte).

17. V. la décision X. c/ RFA (n 1802/62, 26 mars 1963, Annuaire 6, p. 481).

18. CEDH, déc. 28 févr. 2006, Z. et T. c/ Royaume-Uni, précit. 
défendre du sentiment que la Cour souhaite se prémunir contre les dangers d'un impérialisme des droits fondamentaux consistant à exporter sa propre conception de ces droits.

Dans le sillage de cette jurisprudence, la Cour de justice de l'Union européenne a jugé que toute atteinte à la liberté de religion qui viole l'article 10 \& 1 de la Charte des droits fondamentaux ne constitue pas un acte de persécution au sens de l'article $9 \S 1$ de la directive 2004/83 ${ }^{19}$. Cette dernière disposition précise que les actes considérés comme une persécution doivent être « suffisamment graves » en raison de leur nature ou de leur répétition pour constituer une "violation grave des droits fondamentaux de l'homme ", en particulier des droits auxquels aucune dérogation n'est possible en vertu de l'article 15, paragraphe 2, de la Convention européenne. Plus exactement, la Cour a mis en exergue l'importance du critère de gravité des actes ou des risques d'actes de persécution. Chemin faisant, on perçoit un même souci des deux cours européennes de ne pas imposer des charges trop lourdes aux États. L'arrêt de Grande Chambre F.G. c/ Suède, dans laquelle était en cause le refus d'accorder l'asile à un ressortissant iranien converti au luthérianisme après sa fuite en Suède et son expulsion vers l'Iran, ne déroge pas à cette ligne jurisprudentielle puisque la Cour ne se prononce pas sur la violation autonome de l'article $9^{20}$. Critiquable, cette solution l'est en ce qu'elle laisse penser que l'effet extraterritorial de l'article 9 ne pourrait pas être limité par un seuil de gravité. Le juge européen pourrait très bien reconnaître un tel effet en considérant que seules certaines atteintes à la liberté de religion seront prises en considération.

À la lecture de ces affaires, il appert que le raisonnement de la Cour introduit une hiérarchisation entre les droits et libertés garantis par la Convention.

\subsection{L'ESQUISSE D'UNE HIÉRARCHISATION DES DROITS ET LIBERTÉS}

Lorsque l'on s'interroge sur la question d'une éventuelle hiérarchie des droits au sein de la Convention, il est classique d'opposer les droits indérogeables, considérés comme absolus, et les droits conditionnels susceptibles de limitation. À la suite de l'arrêt Soering, il apparaissait que l'effet

19. CJUE, 5 sept. 2012, Bundesrepublik Deutschland c/ Y. et Z., précit., pt. 53.

20. Dans son opinion séparée, le juge Sajo critique le point de vue de la Cour : «J'aurais préféré une analyse séparée de la mesure dans laquelle le droit consacré par la Convention de manifester librement sa religion (c'est-à-dire, en l'espèce, au lieu de dissimuler sa foi chrétienne en Iran, comme l'ont suggéré les autorités nationales) a une application extraterritoriale $»$. 
extraterritorial de la Convention n'était pas sans limites ${ }^{21}$. Exceptionnel, il ne devait concerner que certains droits garantis. La nature du droit en jeu est dès lors une donnée essentielle. La décision $Z$. et T. c/ Royaume-Uni se livre ainsi à de longs développements sur l'importance fondamentale des droits énoncés aux articles 2 et 3. Selon la juge Tulkens, « lorsque l'État tiers n'est lui-même pas partie à la Convention, seules les violations du noyau dur des droits et libertés conventionnels commises par celui-ci sont susceptibles d'engager la responsabilité des États membres ${ }^{22}$. Cette idée d'un " noyau dur» de droits est reprise par Maurice Kamto dans son cinquième rapport sur l'expulsion des étrangers : «Selon le Rapporteur spécial, il paraît irréaliste de prescrire qu'une personne en cours d'expulsion peut bénéficier de l'ensemble des droits de l'homme garantis par les instruments internationaux et par la législation nationale de l'État expulsant. [...] Il semble plus en résonance avec la réalité et la pratique des États de circonscrire les droits garantis durant l'expulsion aux droits fondamentaux de la personne humaine. ${ }^{23}$

Ceci est conforté par l'approche sélective de la Cour. Par exemple, si elle considère qu'une décision d'extradition peut exceptionnellement soulever un problème au cas où l'individu risquerait de subir un déni de justice flagrant dans un État tiers ${ }^{24}$, les droits garantis aux articles 8 et 9 ne bénéficient pas per se de cet effet extraterritorial. En l'état actuel de la jurisprudence, seuls les droits garantis aux articles 2, 3, 5 et 6 bénéficient d'un effet extraterritorial. On le voit, l'extraterritorialité ne découle pas de l'indérogeabilité d'un droit. Le critère distinctif doit dès lors être recherché ailleurs. Comment expliquer que la liberté de religion érigée en "assise d'une "société démocratique" au sens de la Convention » ne bénéficie pas de la même protection? On se souvient que le Professeur Dubouis avait même écrit que « la liberté de religion se situe à un niveau élevé, au sommet peut-être, de la hiérarchie des droits et libertés garantis par la Convention européenne ${ }^{25}$. La hiérarchie des droits

21. "L'article $1[\ldots]$ ne saurait s'interpréter comme consacrant un principe général selon lequel un État contractant, nonobstant ses obligations en matière d'extradition, ne peut livrer un individu sans se convaincre que les conditions escomptées dans le pays de destination cadrent pleinement avec chacune des garanties de la Convention »( 886 , souligné par nous).

22. Tulkens F., « Postface », in Bribosia E. et Hennebel L. (dir.), Classer les droits de l'homme, Bruxelles, Bruylant, 2004, p. 390.

23. Nations unies. Commission du droit international, Cinquième rapport sur l'expulsion des étrangers, présenté par M. Maurice Kamto, Rapporteur spécial, $61^{\mathrm{e}}$ session, Genève, 4 mai-5 juin et 6 juillet- 7 août 2009, A/CN.4/611.

24. Arrêt Soering, § 113. Pour un constat de violation, V. CEDH, 17 janv. 2012, n 8139/09, Othman Abu Qatada c/ Royaume-Uni.

25. Préface de L. Dubouis à la thèse de G. Gonzalez, La Convention européenne des droits de l'homme et la liberté des religions, Paris, Économica, 1997, p. 2. 
revêt parfois dans la Convention une dimension universelle. Aussi, on ne peut écarter totalement l'hypothèse que la question de la mise en jeu de la Convention pour des faits survenus en dehors du territoire des États parties ne peut concerner que des droits dont le caractère universel ne souffre aucune contestation. En ce sens, la jurisprudence Soering serait révélatrice d'un lien entre l'universalité des droits et l'identification d'un noyau dur des droits de l'homme. Relativement à l'interdiction énoncée à l'article 3, la Cour évoque ainsi « une norme internationalement acceptée ». Somme toute, ce n'est ni la fondamentalité du droit, ni son régime juridique qui est déterminant, mais le fait qu'il soit universel. La liberté de religion ne serait pas aussi universelle que le droit à la vie, ou bien encore l'interdiction énoncée à l'article 3. Du reste, conférer un effet extraterritorial à l'article 9 pourrait être vu comme une volonté du juge d'exporter sa conception de la liberté de religion ${ }^{26}$. La Cour le reconnaît d'ailleurs dans l'affaire $Z$. et $T$. en jugeant, à propos de l'article 9, qu'il « s'agit là avant tout de la norme appliquée au sein des États contractants à la Convention, lesquels sont attachés aux idéaux démocratiques, à la prééminence du droit et des droits de l'homme ». Par conséquent, le juge retient pour cette disposition une conception strictement territoriale.

Il n'empêche que le refus de conférer un effet extraterritorial à l'article 9 n'empêche pas la Cour de prendre en compte les risques de persécution religieuse, en particulier lorsqu'elle examine si l'État a respecté ses obligations au titre de l'article 3.

\section{LA PRISE EN COMPTE INDIRECTE DES RISQUES D'ATTEINTE À LA LIBERTÉ DE RELIGION}

Un récent arrêt F.G. c/ Suède, inspiré pour partie de la jurisprudence de la Cour de justice, tend à montrer que le juge européen n'est pas insensible à la situation des demandeurs d'asile qui invoquent un risque de mauvais traitements en raison d'une conversion religieuse. Afin de bien mesurer l'apport de cet arrêt, il sera vu, dans un premier temps, que sa portée n'est

26. La même conclusion s'applique au droit protégé à l'article 8 (CEDH, déc. 22 juin 2004 , $n^{\circ}$ 17341/03, F. c/ Royaume-Uni, et CJUE, 7 nov. 2013, aff. jointes C-199/12 à C-201/12, Minister voor immigratie en Asiel c/ X, Y et Z, V. tout particulièrement le pt. 41 des conclusions de l'avocat général : le but de la directive 2004/83 «n'est pas d'exporter [les droits et libertés garantis par la charte et la CEDH] », "pareille exportation peut en effet être considérée comme une forme d'impérialisme humanitaire ou culturel » (à propos de la reconnaissance du statut de réfugié à des personnes homosexuelles persécutées dans leur pays d'origine). 
pas négligeable sur le terrain des obligations procédurales pesant sur les États (2.1). Puis, dans un deuxième temps, seront examinées les limites du contrôle européen (2.2). Aussi l'arrêt F.G. se prête-t-il à une double lecture.

\subsection{LES AVANCÉES}

C'est essentiellement sur le terrain procédural que la question de la persécution religieuse connaît de réelles avancées. Au cas d'espèce, le requérant soutenait que son éloignement en Iran emporterait violation des articles 2 et 3 de la Convention en raison notamment de sa conversion de l'islam au christianisme en Suède. L'affaire puise son origine dans le refus des autorités suédoises de faire droit à la demande d'asile déposée par le requérant, lequel avait, dans un premier temps, refusé d'invoquer sa conversion à l'appui de sa demande, avant de changer de position lorsque l'arrêté d'expulsion qui le visait est devenu exécutoire. Contrairement à la plupart des affaires concernant la liberté de religion des demandeurs d'asile, le requérant n'a pas fui son pays en raison de persécutions pour des motifs religieux. S'étant converti après son arrivée en Suède, il invoque des risques de persécution liés à cette conversion ${ }^{27}$. Avec des réponses différentes, tant l'arrêt de chambre que celui de la Grande Chambre vont se focaliser sur la prise en compte par les autorités suédoises de la question de la conversion religieuse et de ses conséquences. S'agissant des obligations procédurales pesant sur les États, la Cour rappelle, dans le sillon d'une jurisprudence bien balisée, que dans « le contexte de l'expulsion », " il appartient en principe au requérant de produire des éléments susceptibles de démontrer qu'il y a des raisons sérieuses de penser que, si la mesure incriminée était mise à exécution, il serait exposé à un risque réel de se voir infliger des traitements contraires à l'article 3 ; et que lorsque de tels éléments sont soumis, il incombe au Gouvernement de dissiper les doutes éventuels à ce sujet ${ }^{28}$, en précisant que les demandes d'asile connaissent un régime particulier. Elle a, par exemple, estimé dans l'affaire M.S. c/ Belgique, que lorsque des informations sur un risque général de mauvais traitements sont aisées à vérifier, les autorités doivent évaluer ce risque d'office ${ }^{29}$. Le fait que le demandeur d'asile s'abstienne de soulever l'existence d'un tel risque ne saurait être regardé comme une renonciation

27. Ce qui distingue cette affaire de celle jugée par la Cour de justice de l'Union européenne.

28. $\S 120$.

29. Selon cet arrêt, au moment d'expulser le requérant, les autorités belges savaient ou devaient savoir qu'il n'avait aucune garantie de voir sa demande d'asile examinée sérieusement par les autorités grecques (31 janv. 2012, n 50012/08). 
aux garanties prévues à l'article 3. L'intérêt de l'arrêt F.G. est de préciser que cette obligation s'applique également aux cas où sont invoqués des risques individuels. Alors que, d'ordinaire,

« il incombe à la personne qui sollicite l'asile d'évoquer et d'étayer pareil risque ", il est désormais établi qu'« eu égard toutefois au caractère absolu des droits garantis par les articles 2 et 3 de la Convention, et à la situation de vulnérabilité dans laquelle se trouvent souvent les demandeurs d'asile, si un État contractant est informé de faits, relatifs à un individu donné, propres à exposer celui-ci à un risque de mauvais traitements contraires auxdites dispositions en cas de retour dans le pays en question, les obligations découlant pour les États des articles 2 et 3 de la Convention impliquent que les autorités évaluent ce risque d'office $»^{30}$.

La solution n'était pas complètement prévisible, mais elle ne surprend pas vraiment, car elle s'inscrit dans une tendance visant à renverser la charge de la preuve lorsque le risque de mauvais traitements est de notoriété publique. Il n'est d'ailleurs pas neutre que ce changement intervienne dans une affaire mettant en cause des risques liés à des croyances religieuses. En statuant ainsi, la Cour européenne entend suggérer que la Cour de justice n'a pas le monopole de la protection de la liberté de religion des demandeurs d'asile. Dans leur opinion dissidente commune sous l'arrêt de chambre F.G. c/ Suède, les juges Zupančič, Power-Forde et Lemmens avaient souligné la timidité de la Cour qui semblait faire sien l'argument selon lequel la dissimulation par le requérant de ses convictions religieuses excluait tout risque de mauvais traitements, en comparaison avec l'approche plus protectrice retenue par la Cour de justice dans l'arrêt Bundesrepublik Deutschland c/ Y et Z. La pression des droits fondamentaux a finalement incité la Cour de Strasbourg à revoir sa copie en s'inspirant directement de la jurisprudence de la Cour de justice, peut-être pas la plus pertinente ${ }^{31}$.

Il convient toutefois de ne pas procéder à une approche trop angélique du dialogue judiciaire, la Cour semblant accorder davantage d'importance au caractère absolu des droits garantis par les articles 2 et 3 et à l'extrême

30. § 127. L'arrêt ajoute que « cela vaut spécialement pour les situations où il a été porté à la connaissance des autorités nationales que le demandeur d'asile fait vraisemblablement partie d'un groupe systématiquement exposé à une pratique de mauvais traitements et qu'il y a des motifs sérieux et avérés de croire à l'existence de la pratique en question et à son appartenance au groupe visé ».

31. L'arrêt A (C-148/13), B (C-149/13), C (C-150/13) c/ Staatssecretaris van Veiligheid en Justitie concernait une demande d'asile fondée sur l'homosexualité et l'arrêt Bundesrepublik Deutschland $c / Y$ et $Z$ ne portait pas sur une conversion sur place. 
vulnérabilité des demandeurs d'asile. Reste qu'au moment même où les relations entre les deux cours se sont sérieusement dégradées à la suite de l'avis 2/13 relatif à l'adhésion de l'Union européenne à la Convention, un rapprochement s'opère en faveur de la protection de la liberté de religion des demandeurs d'asile. À preuve, à l'instar de la Cour de justice ${ }^{32}$ et conformément aux positions du Haut-Commissariat des Nations unies pour les réfugiés, la Cour européenne énonce qu'une "personne qui s'est sincèrement convertie ou qui risque de se voir attribuer ses nouvelles convictions religieuses et s'expose ainsi à la persécution ne doit pas être contrainte de cacher sa foi dans le seul but d'échapper à un tel traitement ${ }^{33}$. La Grande Chambre est sur ce point d'une clarté tranchante en désavouant la chambre pour avoir fondé son constat de non-violation sur le fait que le requérant a " cantonné sa foi dans le domaine privé » ${ }^{34}$. Pareillement, toujours dans cette logique «d'absorption ${ }^{35}$ de la liberté de religion par les articles 2 et 3 , la Cour suggère que les hésitations du requérant à invoquer sa conversion religieuse ne sauraient être assimilées à une renonciation des garanties offertes par ces dispositions. À ses yeux, " eu égard au caractère absolu des articles 2 et 3 de la Convention, une renonciation à la protection qui en résulte pour l'individu concerné est peu concevable. Il s'ensuit que, indépendamment de l'attitude du requérant, les autorités nationales compétentes ont l'obligation d'évaluer d'office tous les éléments portés à leur connaissance avant de se prononcer sur l'expulsion de l'intéressé vers l'Iran $»^{36}$.

En définitive, on est conduit à considérer que via les articles 2 et 3 , la liberté de religion fait une entrée remarquée dans le contentieux de l'éloignement

32. «La crainte du demandeur d'être persécuté est fondée dès que les autorités compétentes, au regard de la situation personnelle du demandeur, estiment qu'il est raisonnable de penser que, à son retour dans son pays d'origine, il effectuera des actes religieux l'exposant à un risque réel de persécution. Lors de l'évaluation individuelle d'une demande visant à obtenir le statut de réfugié, lesdites autorités ne peuvent pas raisonnablement attendre du demandeur qu'il renonce à ces actes religieux » (CJUE, 5 sept. 2012, Bundesrepublik Deutschland $c / Y$ et $Z$, précit.).

33. § 145 de l'arrêt F.G.

34. Adde CEDH, 19 déc. 2013, n 7974/11, N.K. c/ France : le requérant doit démontrer qu'il pratique ouvertement cette religion et qu'il est un prosélyte ou, à tout le moins, qu'il est perçu comme tel par les autorités pakistanaises.

35. Gonzalez G., « Conversion sur place et traitement d'une demande d'asile », JCP G 2016, 439.

36. CEDH, Gde ch., 23 mars 2016, F.G. c/ Suède, § 156 (arrêt rendu en matière d'éloignement des étrangers). L'arrêt traduit une évolution par rapport à l'affaire M.S. c/ Belgique dans laquelle la Cour n'estimait pas « devoir se prononcer in abstracto sur le point de savoir si le caractère indérogeable du droit en question entraîne ipso facto l'impossibilité d'y renoncer» (31 janv. 2012, n 50012/08, \& 122). 
des étrangers, au moins sur le terrain procédural. La Cour européenne se montre cependant prudente et ne s'aventure à aucun instant sur le volet substantiel des articles 2 et 3, étape qui aurait permis de transformer l'essai et de donner toute son effectivité à la protection des demandeurs d'asile fondée sur la religion. Au point que cette évolution de la jurisprudence est éclipsée par un autre aspect de l'arrêt, le refus de la Cour de se prononcer sur la violation matérielle des articles 2 et 3.

\subsection{LES LIMITES}

L'arrêt F.G. ne tire pas les conséquences de cette précision de l'obligation procédurale pesant sur les États dans le cadre de l'examen d'une demande d'asile. Alors que la Cour constate in specie que la demande d'asile du requérant fondée sur sa conversion mérite d'être examinée par lesdites autorités, elle élude totalement l'examen du volet substantiel des articles 2 et 3, à savoir les risques encourus par l'intéressé en cas de renvoi vers l'Iran. En bref, elle se satisfait d'un simple constat de violation de l'obligation procédurale dans une situation où une violation potentielle des articles 2 et 3 est clairement établie. Alors certes, la validité de la décision d'expulsion avait expiré, mais dès lors que la Grande Chambre a refusé de radier la requête du rôle, il paraissait logique qu'elle rende un arrêt de principe sur tous les aspects de la question. Une nouvelle fois, elle a paru céder au mouvement de procéduralisation des droits substantiels ${ }^{37}$, moult fois dénoncé en doctrine. Rigoureusement comprise, la démarche suivie implique que le requérant introduise une nouvelle demande d'asile en Suède et que les autorités remédient aux défaillances qui ont entaché la procédure nationale, mais sans que son renvoi soit finalement exclu. La Cour a donc éludé le débat au fond alors que, comme l'ont noté les juges dissidents,

«le requérant risque des poursuites pénales pour crime d'apostasie. Bien que l'État iranien n'ait jamais codifié ce crime, il autorise l'application de certaines lois islamiques alors même que le crime n'est pas spécifiquement mentionné dans le Code pénal [...] dès lors que la répression visant l'acte qui consiste à changer de religion emporte violation du droit à la liberté de religion et contraint en pratique les citoyens musulmans à s'abstenir d'adopter une autre foi. Or, comme l'indique l'article 18 de la Déclaration universelle des droits de l'homme,

37. Dubout E., «La procéduralisation des droits », in Sudre F. (dir.), Le principe de subsidiarité au sens du droit de la Convention européenne des droits de l'homme, Bruxelles, Némésis-Anthémis, 2014, p. 265. 
la liberté de religion implique la "liberté de changer de religion ou de conviction"».

On se souvient que cette formulation avait heurté certains États arabomusulmans lors de l'adoption de la Déclaration universelle des droits de l'homme, l'Arabie saoudite s'étant même abstenue après avoir tenté de faire supprimer le passage de l'article 18 relatif au changement de religion ${ }^{38}$. On fera cependant remarquer que l'apostasie n'a aucun fondement dans le Coran. Plus encore, le verset 256 de la Sourate La Vache «Point de contrainte en matière de religion" et le verset 29 de la sourate La Caverne «Dis : La vérité vient de Dieu, que celui qui veut croire croie, et que celui qui veut être infidèle, le soit » plaident plutôt en sens inverse.

Quand bien même la dernière peine de mort pour apostasie a été exécutée en 1990, celle-ci donne lieu à de nombreuses persécutions et détentions. Semblable retenue judiciaire de la Cour tranche, par exemple, avec ses prises de position sur la question de la compatibilité des peines corporelles prévues en droit musulman avec les exigences de l'article 3. Dans son arrêt Jabari, elle a ainsi jugé que la Turquie ne pouvait expulser la requérante vers l'Iran au motif qu'il existait un risque réel qu'elle subisse la peine de lapidation pour adultère ${ }^{39}$. Il en est de même en ce qui concerne la peine de la flagellation ${ }^{40}$. La sévérité ne serait-elle plus de mise lorsqu'est en cause la liberté de religion? Le moins que l'on puisse dire est que, dans un contexte de recrudescence de l'intolérance religieuse, la position de la Cour fait tache. Il ne faut pas voir dans ces quelques remarques une critique en règle de l'arrêt F.G., à bien des égards innovant. Cet arrêt marque une première étape dans la série jurisprudentielle relative à la prise en compte de la liberté de religion dans le cadre du contentieux de l'éloignement. On ne saurait trop recommander à la Cour de ne pas en rester là...

38. L'article 18 du Pacte international sur les droits civils et politiques a également fait l'objet de nombreuses réserves, V. https://treaties. un.org/Pages/ViewDetails.aspx ? src $=$ TREATY\&mtdsg_no $=$ IV-4\&chapter $=4 \&$ clang $=$ fr\#EndDec $[$ consulté le 13 janv. 2017]

39. CEDH, 11 juill. 2000, $\mathrm{n}^{\circ}$ 40035/98, Jabari c/ Turquie, $\S \S 41-42$.

40. CEDH, 22 juin 2006, $\mathrm{n}^{\circ} 24245 / 03$, D. et autres c/ Turquie, $\S 50$. 